Case Report

\title{
Nonfunctional Metastatic Parathyroid Carcinoma in the Setting of Multiple Endocrine Neoplasia Type 2A Syndrome
}

\author{
María Posada-González, ${ }^{1}$ Joaquín Gómez-Ramírez, ${ }^{2}$ \\ Manuel Luque-Ramírez, ${ }^{3}$ Mercedes Guijarro, ${ }^{4}$ Elena Martín-Pérez, ${ }^{1}$ \\ Ana Rodríguez-Sánchez, ${ }^{1}$ Iñigo García-Sanz, ${ }^{1}$ and Eduardo Larrañaga ${ }^{1}$ \\ ${ }^{1}$ Department of General and Gastrointestinal Surgery, La Princesa University Hospital, 62 Diego de Leon Street, 28006 Madrid, Spain \\ ${ }^{2}$ Department of General and Gastrointestinal Surgery, Fundación Jiménez Díaz Hospital, 2 Reyes Catolicos Avenue, \\ 28040 Madrid, Spain \\ ${ }^{3}$ Department of Endocrinology and Clinical Nutrition, Ramón y Cajal University Hospital, Colmenar Viejo Road 9.100 Km, \\ 28034 Madrid, Spain \\ ${ }^{4}$ Department of Pathology, La Princesa University Hospital, 62 Diego de Leon Street, 28006 Madrid, Spain
}

Correspondence should be addressed to María Posada-González; posada.maria@gmail.com

Received 28 August 2013; Accepted 26 September 2013; Published 20 February 2014

Academic Editors: C. Foroulis, G. Lal, and F. Turégano

Copyright (C) 2014 María Posada-González et al. This is an open access article distributed under the Creative Commons Attribution License, which permits unrestricted use, distribution, and reproduction in any medium, provided the original work is properly cited.

Parathyroid carcinoma is a very rare malignancy. It has been associated with hyperparathyroidism-jaw tumour syndrome, familial isolated primary hyperparathyroidism, and multiple endocrine neoplasia type 1 (MEN-1) and 2A (MEN-2A) syndromes. We report a 54-year-old man with a MEN-2A which presents with a nonfunctional metastatic parathyroid carcinoma and a pheochromocytoma in the absence of medullary thyroid carcinoma. Only a few cases of parathyroid carcinoma have been reported in the literature associated with this syndrome.

\section{Introduction}

Multiple endocrine neoplasia type $2(\mathrm{MEN}-2 \mathrm{~A})$ is a hereditary syndrome characterized by the occurrence of two or more specific endocrine tumours (medullary thyroid carcinoma, pheochromocytoma, or parathyroid adenoma/ hyperplasia). MEN-2A is an autosomal dominant disorder, but each clinical entity is expressed with variable degrees of penetrance. Almost 100\% of patients with MEN-2A develop medullary thyroid carcinoma (MTC). The frequency of pheochromocytoma among these patients is $50 \%$ and of hyperparathyroidism only $10-30 \%$ [1].

Parathyroid carcinoma (PCA) is not a feature of multiple endocrine neoplasia syndromes. In the bibliographic search, only 7 cases of PCA have been described in the setting multiple endocrine neoplasia syndrome type 1 (MEN-1) [2]. To our knowledge, only two cases of parathyroid carcinoma have been reported in a MEN-2A $[3,4]$.
PCA is the rarest endocrine malignancy. More than $90 \%$ of PCA are functional, presenting with symptoms of hyperparathyroidism due to the effects of elevated serum parathormone (PTH) and calcium levels [5]. Nonfunctional PCA are extremely rare, with less than 20 reported cases since 1929 [6].

We present a very unusual case of nonfunctional PCA in the setting of a MEN-2A.

\section{Case Presentation}

We report a 54-year-old man with a past medical history of type 2 diabetes mellitus, obstructive sleep apnoea, and family history of multiple endocrine neoplasia type 2A. The genetic study revealed a heterozygous mutation (Cys618Arg) in exon 10 of the oncogene RET. 


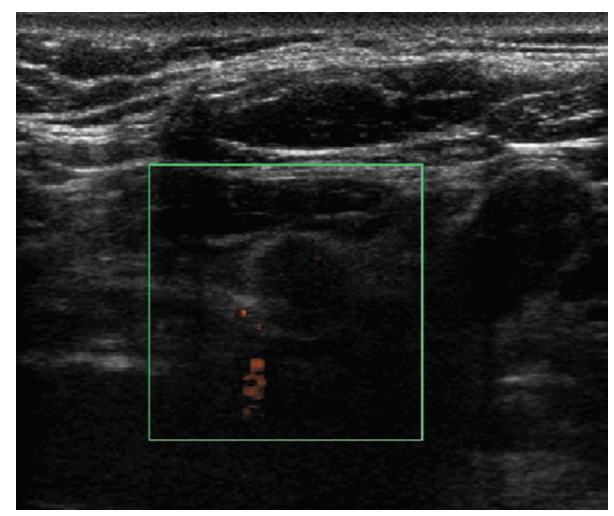

FIgURE 1: Thyroid ultrasound. Hypoechoic nodule in the inferior pole of the left thyroid lobe.

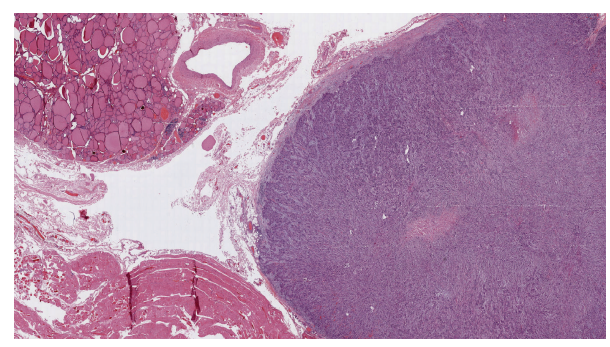

FIgURE 2: Tissue sample. A $1.4 \mathrm{~cm}$ irregularly shaped encapsulated nodular lesion in the inferior pole of the left thyroid lobe which did not depend on the thyroid parenchyma.

He did not have any symptoms and did not refer history of hypertension or paroxysmal hypertensive crises. The serum and urinary catecholamines remained normal. The T2-weighted magnetic resonance imaging showed two subcentimeter-sized lesions in the left adrenal gland, and the 123-I-metaiodobenzylguanidine (MIBG) scan confirmed a functional nodular lesion. A laparoscopic left adrenalectomy was performed, and the final pathology confirmed a pheochromocytoma.

Laboratory tests showed normal calcitonin, calcium, and parathormone serum levels. The thyroid ultrasound showed a hypoechoic nodule in the inferior pole of the left thyroid lobe (Figure 1). The mutation (Cys618Arg) in exon 10 of the oncogene RET is described as a mutation with a higher risk of developing a medullary thyroid cancer, so a prophylactic total thyroidectomy with central lymphadenectomy was performed 3 months after adrenalectomy. The pathological examination showed a $1.4 \mathrm{~cm}$ irregularly shaped encapsulated nodular lesion in the inferior pole of the left thyroid lobe which did not depend on the thyroid parenchyma (Figure 2). In the microscopic examination, we found parathyroid chief cells in trabecular pattern with fibrotic septae, nuclear atypia, and vascular invasion. Immunohistochemical analysis revealed an overexpression of cyclin D1 (Figure 3). A two millimetres lymph node mestastasis with extracapsular invasion was also found (Figure 4). There was no evidence of medullary thyroid carcinoma.

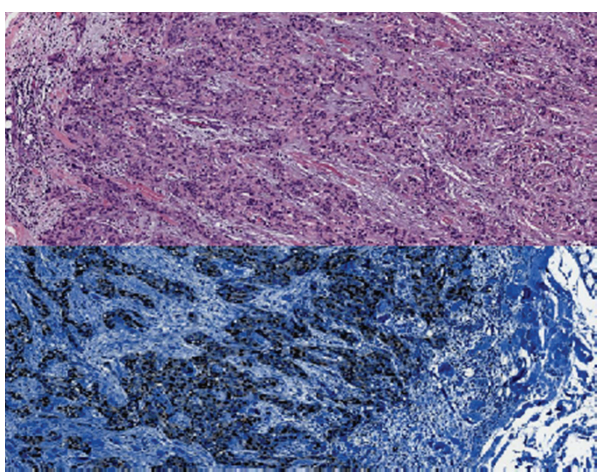

Figure 3: Tissue sample. The microscopic examination of the nodular lesion shows parathyroid chief cells in trabecular, infiltrative pattern with fibrotic septae and vascular invasion. Immunohistochemical analysis revealed a nuclear overexpression of cyclin D1. Cyclin D1 stains the nuclei blue.

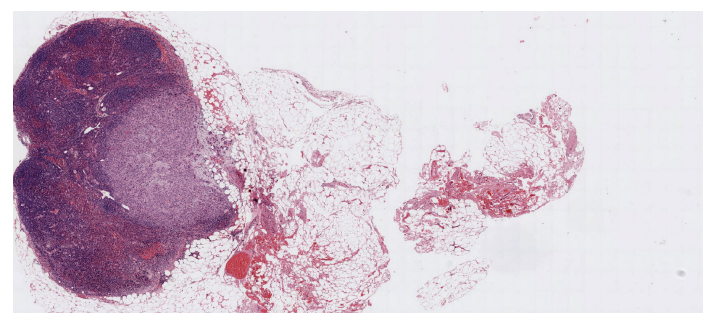

FIgure 4: Tissue sample. Two millimetres lymph node metastasis with extracapsular invasion.

\section{Discussion}

PCA are very infrequent tumors. With an estimated incidence of less than $1 \%$ in all cases surgically treated for primary hyperparathyroidism, they are one of the rarest of all human cancers. According to NCDB reports, their survival at five years is $85.5 \%$ and at ten years is $49.5 \%$. The median age of presentation in most series is between 45 and 60 years. In contrast with primary hyperparathyroidism, which is more common in women (3-4:1), the incidence of PCA is equal between the two genders [5].

The exact aetiology of PCA remains unclear. PCA may appear as a sporadic disease although several genetic defects have also been reported to be involved. Some oncogenes and tumour suppressor genes have been associated to the pathogenesis of PCA. Studies on the tumour suppressor gene CDC73 (formerly HRPT2) have provided the best evidence [7]. CDC73 encodes a protein called parafibromin (parathyroid disease and fibro-osseous lesions). The parafibromin inhibits cyclin D1 activity promoting cellular apoptosis and avoiding cell proliferation. Some studies have shown that mutations of CDC73 may result in a loss of parafibromin expression and, as it is the case of our patient, cyclin D1 overexpression which promotes the neoplastic transformation of the parathyroid tissue [8]. However, more studies are needed to understand the exact role of $\mathrm{CDC} 73$ and parafibromin in the pathogenesis of PCA [9]. 
TABLE 1: Cases of PCA in the setting of MEN-2A reported in the literature.

\begin{tabular}{|c|c|c|c|}
\hline Reference & Jenkins et al. (1997) [3] & J. J. Alfaro (2002) [4] & This case \\
\hline Age (years) & 47 & 49 & 54 \\
\hline Sex & Male & Male & Male \\
\hline RET mutation & (Cys634Tyr) of exon 11 & Not known mutation & (Cys618Arg) of exon 10 \\
\hline Clinical presentation & 8-week symptoms of thirst and polyuria & Severe hypercalcemia & Asymptomatic \\
\hline Serum calcium $(\mathrm{mmol} / \mathrm{L})^{*}$ & 3.3 & 3.77 & 2.3 \\
\hline Serum PTH $(\mathrm{pmol} / \mathrm{L})^{\circ}$ & 102 & 1399 & 6.1 \\
\hline Serum calcitonin $(\mathrm{ng} / \mathrm{L})^{6}$ & 5110 & 75.8 & 2.1 \\
\hline Serum and urinary metanephrines & Normal & Normal & Normal \\
\hline Tumour size $(\mathrm{cm})$ & Not recorded & Not recorded & 1.4 \\
\hline Metastases & First thoracic vertebra (T1) and right frontal bone & Lung & Cervical lymph node \\
\hline Medullary carcinoma & Yes & Yes & No \\
\hline Pheochromocytoma & No & No & Yes \\
\hline
\end{tabular}

* Serum calcium normal limit $(2.1-2.5 \mathrm{mmol} / \mathrm{L})$.

${ }^{\circ}$ Serum PTH normal limit (11-54 ng/L).

${ }^{6}$ Serum calcitonin normal limit (males $<13.8 \mathrm{ng} / \mathrm{L}$; females $<6.4 \mathrm{ng} / \mathrm{L}$ ).

There are also some other clinical situations such as external radiation neck exposure or end-stage renal disease, which may predispose to the development of PCA. In addition, PCA has been associated with familial isolated primary hyperparathyroidism, hyperparathyroidism-jaw tumour syndrome, MEN-1, and MEN-2A [6].

Apart from our patient, there are just two cases of PCA in the setting of MEN-2A reported in the literature. Details of the cases are displayed in Table 1.

More than $90 \%$ of PCA are functional, presenting with symptoms of hyperparathyroidism due to effects of elevated serum parathormone $(\mathrm{PTH})$ and calcium levels. Malignancy is generally related to calcium levels greater than $3.5 \mathrm{mmol} / \mathrm{L}$ and PTH levels more than 5 times the upper limit of the normal range. Nonfunctional PCA are extremely rare. They account for approximately $1.9 \%$ of parathyroid tumours with less than 20 reported cases since 1929 [6,10]. As in our patient, nonfunctional PCA remain normocalcemic with normal serum levels of PTH.

Most of the reported normocalcemic PCA cases present with locally advanced neck masses. This may be due to the fact that they are larger at presentation because of the absence of clinical manifestations. They also show frequent locoregional metastasis and recurrence. Despite the fact of the short number of nonfunctional PCA cases described and the difficulty to compare them to patients with functional PCA, nonfunctional PCA seem to be more aggressive tumors representing a poor prognosis factor [10].

The treatment of PCA is based on the complete surgical removal of the tumors including adjacent tissues involved. This is the only curative treatment which provides the best long-term survival. Nonsurgical therapies such as chemotherapy and radiation have shown poor results and their use in the PCA treatment is still not well defined [9].

The diagnosis of PCA requires a histological confirmation. However, there is a big difficulty to distinguish between the histopathological features of benign and malignant parathyroid tumors. In 1973, Schantz and Castleman published the histological criteria for the diagnosis of PCA [11]. These classical histological features which define PCA are the presence of capsular or vascular invasion, trabeculated fibrous parenchyma, and mitotic figures. Unfortunately, these features can also appear in benign parathyroid tumours, so this is why the capsular or vascular invasion are considered to be the most specific features of PCA [12]. Some immunohistochemical markers have been used to try to improve accuracy in identifying parathyroid malignancy. As we have already indicated, overexpression of cyclin D1 seems to be a specific feature of PCA. As in our patient, it has been identified in a high percentage of parathyroid carcinomas. In 1999, Vasef et al. presented evidence that cyclin D1 is highly expressed in PCA specimens (91\%) compared with parathyroid adenomas (39\%) [13].

Our patient represents a very unusual case, and not only because of the presence of PCA associated to a MEN-2A syndrome. It is also remarkable that our patient shows no evidence of MTC, even though the penetrance of MTC in the MEN-2A syndrome is almost $100 \%$. Nowadays and thanks to all studies developed during the last years, some mutationspecific risk profiles (genotype-phenotype correlation) have been established. The mutation of the RET proto-oncogene found in our patient (Cys618Arg) shows a high risk to develop a MTC [14]. However, the histopathological study after the thyroidectomy revealed no signs of MTC.

We must conclude that this patient may be representing a variation from the classical multiple endocrine neoplasia syndrome type $2 \mathrm{~A}$ which develops a pheochromocytoma and a parathyroid carcinoma in the absence of medullary thyroid carcinoma.

\section{Conflict of Interests}

The authors declare that there is no conflict of interests regarding the publication of this paper. 


\section{References}

[1] N. Wohllk, H. Schweizer, Z. Erlic et al., "Multiple endocrine neoplasia type 2," Best Practice and Research, vol. 24, no. 3, pp. 371-387, 2010.

[2] C. del Pozo, L. García-Pascual, M. Balsells et al., "Parathyroid carcinoma in multiple endocrine neoplasia type 1. case report and review of the literature," Hormones, vol. 10, no. 4, pp. 326331, 2011.

[3] P. J. Jenkins, M. A. Satta, M. Simmgen et al., "Metastatic parathyroid carcinoma in the MEN2A syndrome," Clinical Endocrinology, vol. 47, no. 6, pp. 747-751, 1997.

[4] J. J. Alfaro, C. Lamas, J. Estrada, and T. Lucas, "MEN-2A syndrome and pulmonary metastasis," Postgraduate Medical Journal, vol. 78, no. 915, pp. 51-52, 2002.

[5] J. M. Sharretts, E. Kebebew, and W. F. Simonds, "Parathyroid cancer," Seminars in Oncology, vol. 37, no. 6, pp. 580-590, 2010.

[6] B. J. Wilkins and J. S. Lewis Jr., "Non-functional parathyroid carcinoma: a review of the literature and report of a case requiring extensive surgery," Head and Neck Pathology, vol. 3, no. 2, pp. 140-149, 2009.

[7] A. Diggonet, A. Carlier, E. Willemse et al., "Parathyroid carcinoma: a review with three illustrative cases," Journal of Cancer, vol. 2, pp. 532-537, 2011.

[8] C. Zhang, D. Kong, M. H. Tan et al., "Parafibromin inhibits cancer cell growth and causes G1 phase arrest," Biochemical and Biophysical Research Communications, vol. 350, no. 1, pp. 17-24, 2006.

[9] S. H. Fang and G. Lal, "Parathyroid cancer," Endocrine Practice, vol. 17, supplement 1, pp. 36-43, 2011.

[10] C. L. Messerer, S. P. Bugis, C. Baliski, and S. M. Wiseman, "Normocalcemic parathyroid carcinoma: an unusual clinical presentation," World Journal of Surgical Oncology, vol. 4, article 10, 2006.

[11] A. Schantz and B. Castleman, "Parathyroid carcinoma. A study of 70 cases," Cancer, vol. 31, no. 3, pp. 600-605, 1973.

[12] P. K. Mandal, S. Ray, and N. Basu, "Parathyroid carcinoma uncovering the enigma: case report and review of literature," Journal of Cytology, vol. 28, no. 4, pp. 223-225, 2011.

[13] M. A. Vasef, R. K. Brynes, M. Sturm, C. Bromley, and R. A. Robinson, "Expression of cyclin D1 in parathyroid carcinomas, adenomas, and hyperplasias: a paraffin immunohistochemical study," Modern Pathology, vol. 12, no. 4, pp. 412-416, 1999.

[14] F. Raue and K. Frank-Raue, "Genotype-phenotype relationship in multiple endocrine neoplasia type 2. Implications for clinical management," Hormones, vol. 8, no. 1, pp. 23-28, 2009. 


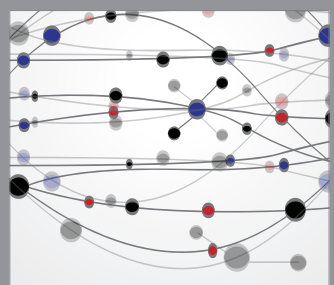

The Scientific World Journal
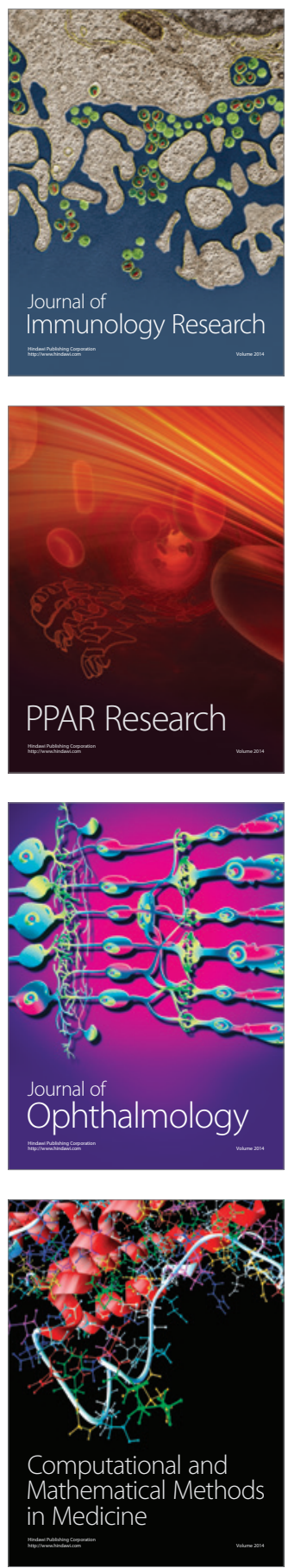

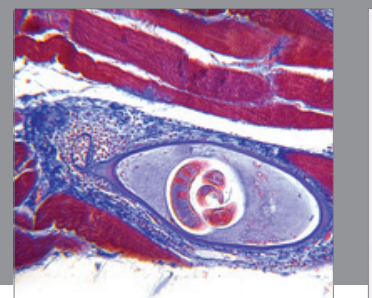

Gastroenterology

Research and Practice
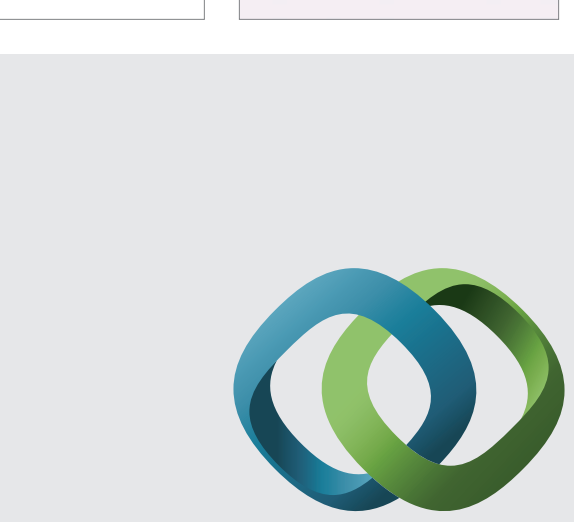

\section{Hindawi}

Submit your manuscripts at

http://www.hindawi.com
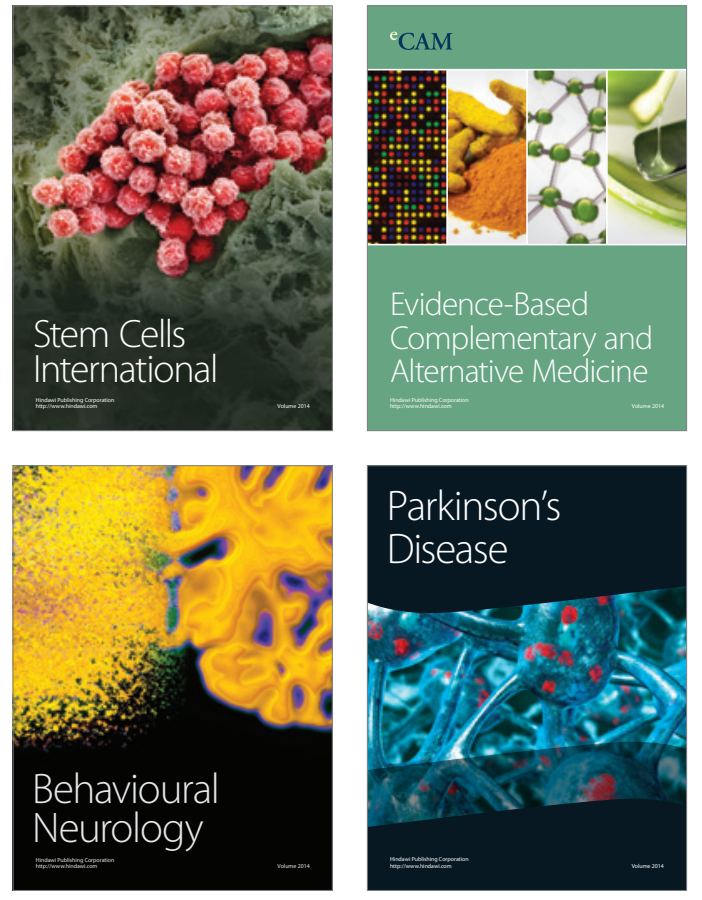
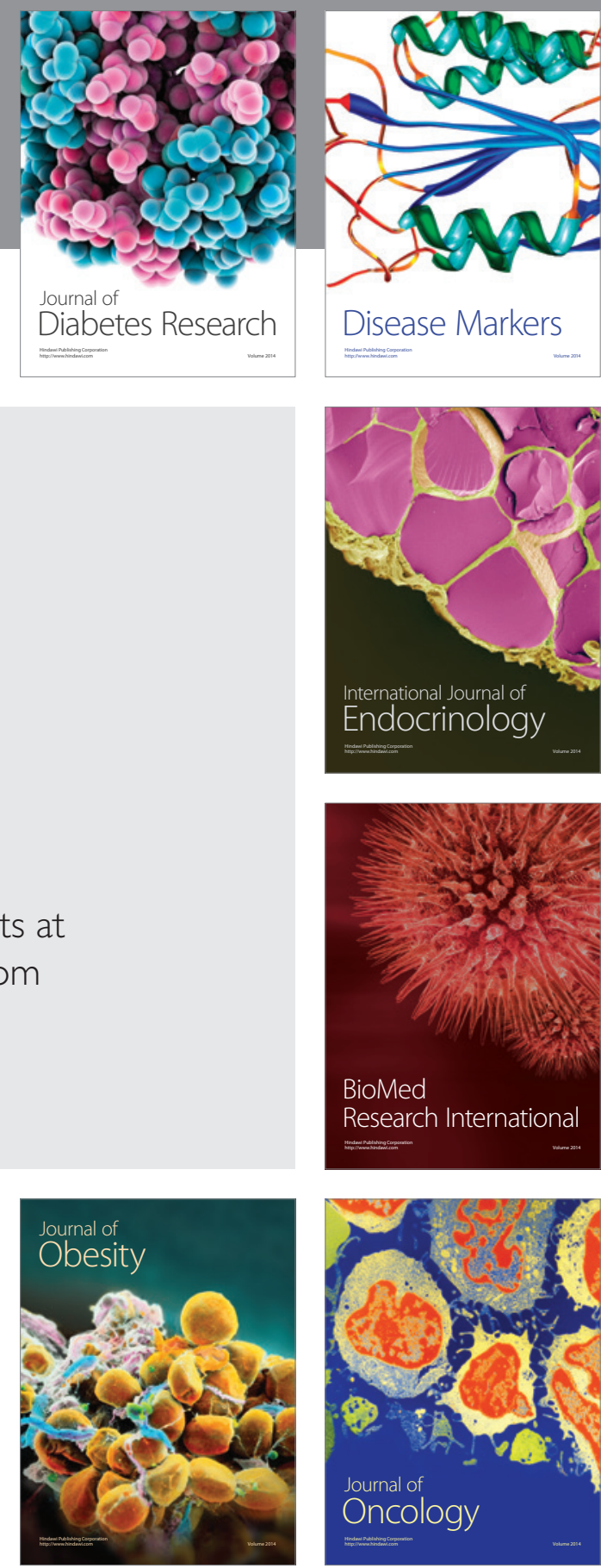

Disease Markers
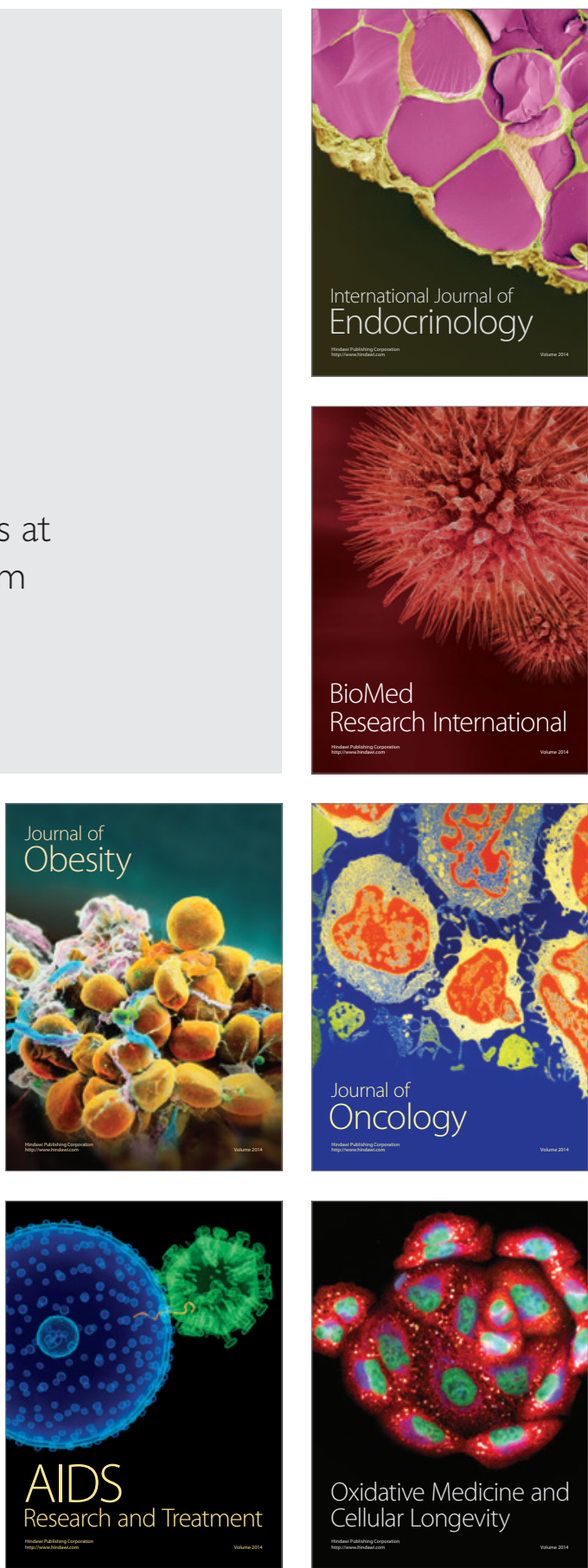